\title{
A Speculative Computation Approach for Conflict Styles Assessment with Incomplete Information
}

\author{
Marco Gomes, Tiago Oliveira and Paulo Novais \\ Department of Informatics, University of Minho, Braga, Portugal \\ \{marcogomes, toliveira, pjon\}@di.uminho.pt
}

\begin{abstract}
This paper analyses a way to cope with incomplete information, namely information regarding the conflict style used by parties. This analysis is important because it enables us to develop a more accurate and informed conflict style classification method to promote better strategies. To develop this proposal, an experiment using a combination of Bayesian Networks with Speculative Computation is depicted. Thus, in this work, was firstly identified and applied a set of methods for classifying conflict styles with incomplete information; secondly, the approach was validating opposing data collected from a web-based negotiation game. From the experiment outcomes, we can concluded that it is possible to cope with incomplete information by producing valid conflict style default values and, particularly, to anticipate competing postures through the dynamic generation of recommendations for a conflict manager. The findings suggest that this approach is suitable for handling incomplete information in this context and can be applied in a viable and feasible way.
\end{abstract}

Keywords: Intelligent System, Speculative Computation, Conflict Styles

\section{Introduction}

In a conflict resolution process, parties may assess the same situation in different ways, and, as such, respond differently. It is, therefore, necessary to understand the conflict (response) styles of the parties involved so as to manage conflicts properly. Another important step in this process is the one of the actual negotiation between the parts. A step in which they have to come up with concrete and valid outcomes, which may be rejected or probably changed by the other part [1]. A basic ingredient of this process is the correct anticipation of the parties' actions. In this sense, and whatever specific conflict style is used to solve or to manage the conflict, both parties may be attempting resolution of the conflict by acting based on their individual perception of the situation. However, a perception based on the faulty or incomplete information could undermine the resolution process and can lead to erroneous conclusions and therefore to an inexact evaluation of consequences. And this, surely, can be problematic for parties or a conflict manager who do not have a complete picture of each other's behaviours.

Therefore, a cornerstone of this approach is the idea that if a conflict manager, in an intelligent conflict support system, has the knowledge to be able to identify parties' conflict styles he will promote better strategies and, consequently, lead to better 
solutions. Our work is, hence, devoted to investigations on how to cope with incomplete information when analysing the parties' conflict styles. Additionally, this kind of analysis is critical to enable us to develop a more accurate and informed conflict style classification method. Furthermore, this work describes the design of a speculative computation framework to cope with incomplete information regarding the parties' conflict style assessment. In general terms, this approach can be seen as a tentative procedure when complete information about conflict style is not obtained. Although the following terms may be associated with different meanings, the terms "conflict style" and "negotiation style", in this section and throughout this study, are used interchangeably. The remainder of this paper is structured as follows. Section 2 presents a brief review of a conceptual framework to facilitate information gathering in a municipal conflict support system. Further, the means by which the conflict style is classified in a digital environment are depicted. A case scenario is presented. In Sections 3 and 4, we describe our approach to improving the classification of conflict styles with incomplete information in a generic municipal decision-making process. The final section details the main conclusions drawn from this study.

\section{Municipal Intelligent Conflict Support System}

The proposed system is a conceptual framework which can be implemented as a computerbased support system to assist municipal decision-making when handling conflict. The system consists of components underpinning an intelligent environment that could be sensitive and responsive to both parties of a conflict management process. Pragmatically speaking, the system was designed to sense conflict context, acquire it and then make reasoning on the acquired context and thus acting on the parties' behalf. On the one hand the system builds up a consented information database of each and can link that subsequently with the proper individual performance within the conflict process that is monitored by the system. On the other hand, while the user conscientiously interacts with the system and takes his/her decisions and actions, a parallel and transparent process takes place in which contextual and behavioural information is sent in a synchronized way to the conflict support platform. Upon converting the sensory information into useful data, the platform allows conflicting manager for a contextualized analysis of the user's data. It is out of the scope to explain in detail each component of the system. Despite that, regarding the main aim of this work, the way how conflict styles are assessed in a digital environment is depicted as follows.

\subsection{Conflict Style Assessment Model}

Conflicts can develop in stages and consequently may involve many different responses as the conflict proceeds. People involved develop various strategies, solutions or behaviours, to deal with the conflict. The style of managing a conflict that each one has must be seen as having a preponderant role in the outcome of a conflict resolution process, especially on those in which parties interact directly (e.g., negotiation, mediation). To classify the conflict style, the proposals must be analysed, namely regarding their utility. In that sense, in each stage of the negotiation the parties' proposals are analysed 
according to their utility value and a range of possible outcomes defined by the values of the Worst Alternative to a Negotiated Agreement (WATNA) and Best Alternative to a Negotiated Agreement (BATNA) of each party. This approach uses a mathematical model [2], which classifies a party's conflict style considering the range of possible outcomes, the values of WATNA and BATNA as boundaries, and the utility of the proposal. Regarding that utility, it quantifies how good a given outcome is for a party, it is acceptable to argue that a competing party will generally propose solutions that maximize its own utility in expense of that of the other party (the utility of the proposal is higher than the WATNA of the other party), whereas, for example, a compromising party will most likely search for solutions in an intermediary region (the utility of the proposal falls within the range of the zone of possible agreement, the range of overlapped outcomes that would benefit both parties). Essentially, we were able to classify the personal conflict style of a party by constantly analysing the utility of the proposals created. Once the styles are identified, strategies can be implemented that aim to improve the success rate of procedures for resolution and conflict management.

\subsection{Municipal Service Delivery - Proof of Concept}

The proof of concept was chosen to demonstrate the applicability of the concepts underpinning this study. Employing this method, it aimed to prove the viability and feasibility of innovative concepts through prototypes and demonstrations of features. In this case, the proof was designed purely to demonstrate the functionality of Speculative Computation approach to deal with incomplete information regarding the classification of conflict styles. The broader aim is to experiment a path of research in which innovative methods to manage conflict could be integrated into a generic municipal decisionmaking process. This first attempt at performing something that might be real-world usable comprises the definition of a scenario, a simulation environment and analysis of the outcomes.As stated before, for the purpose of simulating a conflict situation in reallife municipal environments a previous developed web-based simulation was adapted to this purpose. It was adapted to enable test participants having a conflict experience induced by the presence of an Ambient Intelligence system. In that sense, a game was designed to simulate a scenario in which a municipality needs to perform a service contract (an agreement between a municipality and a service provider) to guarantee the repairs and maintenance of municipal equipment. Each party has to achieve a desired result in the negotiation, in other words, the negotiation outcome was to be a win/win situation for both sides. The game starts with the application randomly giving one of the predetermined roles to each party. The instructions to win the game were to negotiate a fair deal and make sure that the party in question did not miss the deal. Each party's instructions were clearly presented, visible to them through the application interfaces. To classify the conflict style, the proposals must be analysed regarding their utility. In each stage of the negotiation, the parties' proposals are analysed according to their utility value and a range of possible outcomes defined by the values of the worst alternative to a negotiated agreement (WATNA) and best alternative to a negotiated agreement (BATNA) of each party. Regarding the conflict style analysis, the ZOPA (Zone Of Possible Agreement) was bounded by the BATNA (10000 Euro) and WATNA (12000 Euro) values. The range of possible agreement was 2000 , but the parties were not aware of this 
detail. The participants of the proposed experiment were volunteers socially connected with our lab members. Forty individuals participated, both female and male,aged between 22 and 53. During the experiments, the information about the user's context was provided through a monitoring framework, which is customized to collect and treat the interaction data. The participants played the web-based game through computers that allowed the analysis of the described features. The obtained results comprise data about negotiation experiments carried out in a controlled environment in our lab. The results will be analysed in following sections.

\section{A Probabilistic Model for Conflict Style Assessment}

The key issue to using a Speculative Computation approach to cope with incomplete information in a negotiation, namely information regarding the negotiation style used by participants, is to be able to generate a likely set of default parameters. The same is to say, to be able to predict the negotiation styles of participants in each round. The intuition here is that the conflict style exhibited by a participant in a round will affect the conflict style used by the very same participant in the following round. A predictive model capable of this has to produce the most likely values for a set of parameters, in this case for the negotiation style and establish dependence relationships between the negotiation styles in different rounds. Networks (BNs), for their set of characteristics [3], fulfil these requirements.

\subsection{Bayesian Networks}

BNs are graphical representations of statistical dependences and independences between variables [3]. They provide a network structure and a probability distribution that are easily interpreted by humans and machines. The problem at hand goes beyond the typical classification and regression problems. It is, instead, a density estimation problem where the objective is to find the most likely collective state of information for the negotiation styles in successive rounds of negotiation. As such, BNs fit the problem description better than other machine learning models [3]. Formally, a BN is an acyclic directed graph $G=\left(V(G), A(G)\right.$ with a set of vertices $V(G)=\left\{V_{1}, \ldots, V_{n}\right\}$ where each vertex $V i \in V(G)$ represents a discrete stochastic variable, and a set of arcs $A(G) \subset V(G) X V(G)$ where each $\operatorname{arc}\left(V_{i}, V_{j}\right) \in A(G)$ represents a statistical dependence. A BN defines a joint probability distribution $\operatorname{Pr}$ that may be factorized according to Equation 1, where $\pi$ is associated with the set of variables that denote the vertex parents of $V_{i}$.

$$
\operatorname{Pr}\left(V_{i}, \ldots, V_{n}\right)=\prod_{i=1}^{n} \operatorname{Pr}\left(V_{i} \mid \pi\left(V_{i}\right)\right)
$$

\subsection{Building the Predictive Model}

Since previous analyses [4] revealed that the role of a participant significantly influences his negotiation style, Sellers and Buyers should be modelled in different networks. Understanding the dependence relationships between negotiation styles in different rounds 
is possible by performing $\mathrm{BN}$ structure learning on the data gathered from the experiments. Some of the most well known BN structure learning algorithms include: the hill-climbing $(h c)$ search [5], the grow-shrink ( $g s)$ [5], the max-min hill-climbing $(m m h c)$ and restriction maximization (rsmax2) [6], and the chow-liu algorithm. Using the above-mentioned algorithms, the goal is to obtain the network structure that best describes the dependences between negotiation styles in different rounds, based on the available data. Different scores can be used to assess the fitness of the network. In the present work, the Akaike Information Criterion (AIC) [7] was the selected metric. It is defined as in Equation 2.

$$
A I C=\log L\left(X_{1}, \ldots, X_{n}\right)-d
$$

The $\log L$ component corresponds to an assessment of goodness of fit, of how well a model fits the data. The $d$ component is a penalty that is an increasing function of the number of estimated parameters and is used to avoid overfitting. Given Equation 2, the goal is to maximize the AIC. With a BN structure and the corresponding probability distribution, it is possible to retrieve the values for the variables that maximize the distribution, given available evidence. This is modelled as a Maximum a Posteriori estimation (MAP), defined as in Equation 3 [8].

$$
M A P_{\theta}=\underset{\theta}{\operatorname{argmax}} \operatorname{Pr}(\theta \mid e)
$$

The $\theta$ component represents the goal variables for which the estimation is calculated and $e$ represents the available evidence. This will be the mechanism by which the default values for negotiation styles in successive rounds of negotiation will be produced. These values will be used in a Speculative Computation framework that models interventions in the negotiation in order to influence the participants to adopt collaborating negotiation styles.

\subsection{Determining Default Values for Conflict Styles}

The above-mentioned experiments produced data regarding 20 negotiation games, which means that there were data for 20 Sellers and 20 Buyers. Out of all the games, the minimum number of rounds was two, the maximum was 12, and the most frequent number of rounds was three (observed in nine games). It was also possible to observe that $95 \%$ of the games had three or more rounds. Given this analysis it was decided that the model would feature predictions up to three rounds of negotiation. As such, two data sets were produced, one for Sellers and another for Buyers. Each data set had three columns representing the first three rounds, filled in with the negotiation styles exhibited by 19 participants for each data set. Only the records of the game with two negotiation rounds were excluded.The structure learning algorithms mentioned in the previous section were applied to the two data sets, producing the AIC scores shown in Table 1.

At the same time, a network structure was modelled based on the underlying intuition for the work, i.e, that the negotiation style exhibited in a round, by both Sellers and Buyers, would be probabilistically dependent on the negotiation style exhibited in 
Table 1: Results of the structure learning and structure assessment processes.

\begin{tabular}{|c|c|c|}
\hline \multirow{2}{*}{ Network Model } & Seller & Buyer \\
\cline { 2 - 3 } & AIC score & AIC score \\
\hline hc & -38.749 & -78.361 \\
\hline gs & -40.233 & -78.361 \\
\hline mmhc & -40.233 & -78.361 \\
\hline rsmax2 & -40.233 & -78.361 \\
\hline chow-liu & -40.795 & -79.990 \\
\hline expert & -40.795 & -79.990 \\
\hline
\end{tabular}

the previous round. Let Round1,Round 2 , and Round 3 be variables that represent the negotiation styles in rounds 1,2 , and 3 , this supposition can be translated into the network structure. The network was called expert. In Table 1, it is possible to observe that the AIC scores of the network structures were, overall, very low. This was most likely due to the reduced size of the sample used to learn them. However, given the complex data collecting process described above, the experiments would have had to be carried out on a much larger scale in order to produce better results. That being said, based on the available scores, the best network structure for the Seller role was the one resulting from the $h c$ algorithm. The network is shown in Figure 1a and its score was even higher than the expert network structure, thus contradicting the initial intuition. It has a diverging arc disposition and the negotiation styles of Round 2 and Round 3 are shown to be conditionally independent given Round 1 . Throughout the rounds, it is possible to observe that Sellers remain competitive, but the probability of their assuming a collaborating negotiation style does increase. Regarding the networks for the Buyer role, the $h c, g s, m m h c$ and rsmax 2 algorithms produced the same type of structure, which was also, coincidentally, the highest scoring network. It is presented in Figure 1b. Again, the score was higher, although slightly, than that of the expert network. It was only possible to establish a probabilistic dependence relationship between the negotiation styles of Round 1 and Round 2 . Round 3 was considered to be independent from the other two. Be that as it may, the network reveals that Buyers usually start with a competing negotiation style, but they are the ones to respond to the lack of dynamism in the negotiation by changing the negotiation style towards accommodating in the third round, thus unlocking a possible impasse.

With the networks of Figure 1 and using MAP queries it is possible to obtain the most likely negotiation styles for the three rounds before the negotiation starts, or midway, for the remaining rounds, after a round is over. Supposing one is at the beginning of the negotiation, no round has taken place yet, and there is no information about previous negotiation styles, it would be possible to determine the most likely set of negotiation styles of a Seller for each round. Performing the MAP query with no evidence on the network of Figure 1a would result in assuming, by default, that Round1, Round 2 , and Round 3 would have the value competing, with a probability of 0.563 . Now, supposing the first round is over and the Seller adopted a collaborating style instead, the MAP query to determine the most likely styles in the next two rounds would have Round $1=$ collaborating as evidence. This query would produce two sets of predictions. 


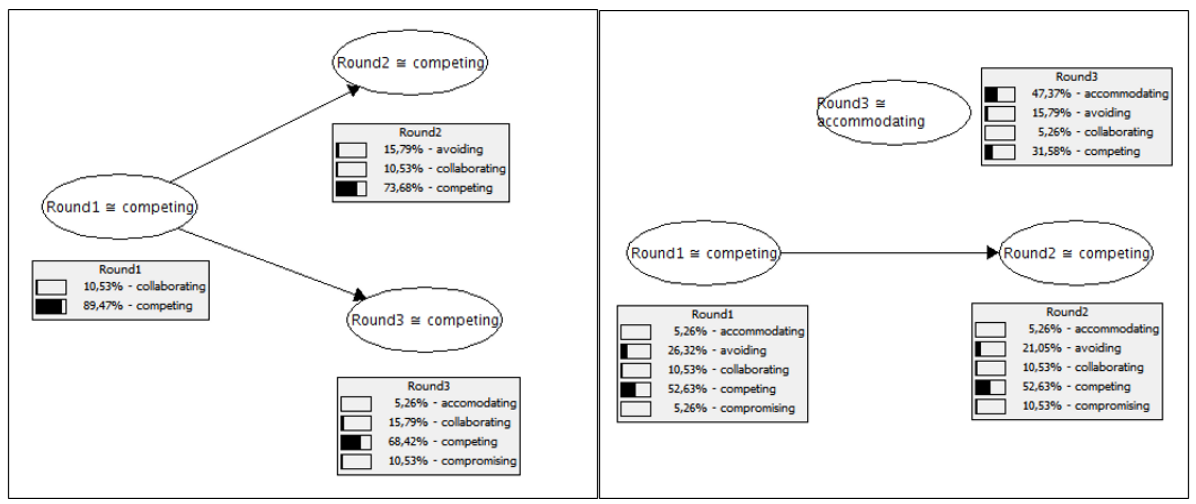

(a) Selected network structure for Sellers

(b) Selected network structure for Buyers

Fig. 1: Bayesian network structures.

The first would be Round $2=$ collaborating and Round $3=$ collaborating, and the second would be Round $2=$ collaborating and Round $3=$ acommodating. Both sets would have a probability of 0.500 . These predictions are used in the Speculative Computation framework modelling the interventions of the conflict manager in the negotiation.

\section{Speculative Computation Approach to Cope with Incomplete Information}

The part taken by the conflict manager in the negotiation is that of trying to bring it to a satisfactory conclusion to both parties, to make them collaborate. As such, it has to anticipate the negotiation style used by the participants in each round, fill in this incomplete information, and recommend changes to the behaviours of the participants. This is modelled using Speculative Computation.

\subsection{Speculative Computation Framework}

Speculative Computation is a logic programming framework that structures reasoning with incomplete information, based on the use of default values. Its complete semantics and procedures are presented in $[9,10]$. The framework has two principal components:

- Default set $(\Delta)$ : This is a set containing default values for the parameters that serve as premises in the inference process;

- Logic Program $(\mathscr{P})$ : This is a set of rules that allow the drawing of conclusions based on specific parameters;

The normal execution of the framework includes providing values for the parameters and traversing the rules in $\mathscr{P}$ until a conclusion is reached. The execution is structured in three phases: 
- Process Reduction Phase: This corresponds to the derivation of tentative conclusions based on the existing values for the inference parameters (defaults or not). At the start of the computation all the values are default values. This results in likely scenarios. The computations that are not consistent with the defaults are suspended, but can be activated in a later phase;

- Fact Arrival Phase: This corresponds to the replacement of a default value for a parameter with the true observed value. As a result, the scenarios produced in a previous phase are revised. Those that are still consistent are maintained and those that become inconsistent are removed. At the same time, suspended computations can be resumed and become new scenarios;

- Default Revision Phase: This corresponds to the replacement of a default value for a parameter with a new default value. This new default value may be produced by the arrival of a true observed value that conditions another parameter. As a result, the scenarios produced in a previous phase are revised.

Speculative Computation fits systems that have a well-defined logic. It can serve as an interface between a predictive model and the rules that guide the enactment of system functions. Its advantage is in the clear definition of the procedures to manage the derived conclusions in a logic framework, according to the ever-changing state of the information.

\subsection{Generating Recommendations for the Conflict Manager}

The main procedures occurring in the conflict manager assume the form of Speculative Computation phases. The worflow of the conflict manager is depicted in Figure 2.

The set of rules that allow the inference of the recommendations the conflict manager may provide to Sellers or Buyers, based on their negotiation styles, is represented in the $\mathscr{P}$ component of the framework. At the beginning of the negotiation, when there is no information about the negotiation styles, a MAP query, such as the one performed in Section 3.3, provides the initial default set $\Delta$. As shown in Figure 2, through a process reduction phase this would result in tentative scenarios representing possible recommendations of the conflict manager. For instance, in the example given above, it was ascertained that the most likely negotiation style of a Seller in the first three rounds would be competing. Competing would be then used in $\Delta$ for the three rounds, and process reduction would produce a scenario in which the conflict manager should advise the Seller to lower the value of his proposal, in order to become more collaborating in the first round. After the first round actually takes place, the true negotiation style is observed and it triggers a fact arrival phase. The previous scenarios are revised and incorporate the newly arrived information. At the same time, the observed negotiation style is used to condition the Bayesian probabilistic model with a new MAP query, which results in a new default set $\Delta$. This new default set is used in the default revision phase to generate a new set of scenarios and, thus, new recommendations for the conflict manager to give. Supposing the observed negotiation style of the Seller in round one was collaborating, as an effect of the recommendation provided by the conflict manager, this would be used to condition parameter Round 1 in the Seller BN, 
through the second MAP query given as an example in Section 3.3. This would generate a new default set with the style collaborating for round two and collaborating or accommodating for round three. With default revision, this new default can be used to produce scenarios that let the conflict manager know that it should not intervene since predicted negotiation style is according to the goal it set out for the Seller. This whole process repeats itself throughout the ensuing rounds until the negotiation is brought to a conclusion.

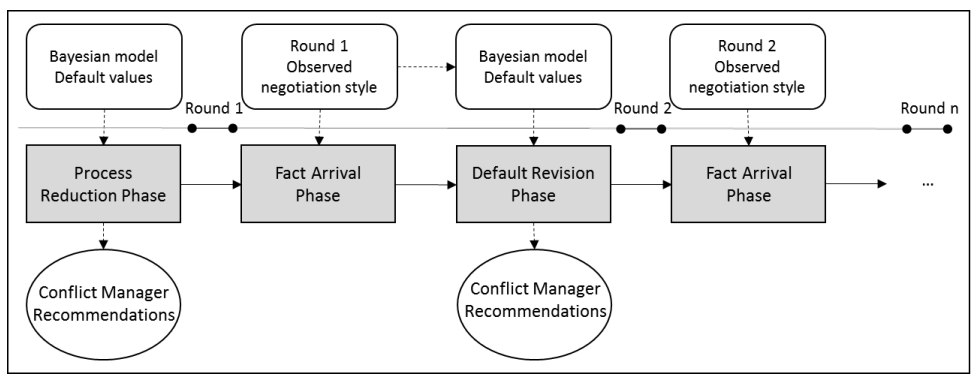

Fig. 2: Workflow carried out by the Conflict Manager based on a Speculative Computation framework.

The goal here is to model a dynamic and flexible conflict manager. For this purpose, BNs are combined with Speculative Computation. While BNs have the task of producing default values, Speculative Computation structures the reasoning process. This allows a quick adaptation to changes in the negotiation style throughout the rounds. This setting also provides a mechanism for the management of conflicts through the anticipation of competing postures.

\section{Conclusion}

This study set out to determine a way to deal with incomplete information, namely information regarding the conflict style used by parties. Thus, firstly we aim to identify and apply a set of methods for classifying conflict styles with incomplete information; secondly, we aim to validate this approach opposing data collected from a web-based negotiation game. The findings suggest that in general it is now possible to state that our approach copes with incomplete information by producing valid conflict styles default values; and anticipating competing postures through the dynamic generation of recommendations for a conflict manager. So, facing these results, we can claim that this work advances the state of the art in that such an analysis has not previously been undertaken. Meanwhile, due to the small sample size used in the current study, some caution must be taken when interpreting the results of the statistical analysis presented and underpinning the conclusions. Additionally, limitations of the current research must be pointed out. First, the participants were recruited from a particular population- a population that 
may limit the generalizability of the results. Admittedly, the participants of the experiment may not be representative of conflict parties in general. Consequently, we are unable to demonstrate the causality of the variables conclusively. Finally, returning to the question posed at the beginning of this study, it is now possible to state that is possible to cope with incomplete information to enable a more accurate characterization of conflict styles in a resolution process. However, more research is needed to provide more definitive evidence.

\section{Acknowledgements}

This work has been supported by COMPETE: POCI-01-0145-FEDER-007043 and FCT - Fundação para a Ciência e a Tecnologia (Portuguese Foundation for Science and Technology) within the Project Scope UID/CEC/00319/2013.

\section{References}

1. Andrew Stranieri and John Zeleznikow. Tools for intelligent decision support system development in the legal domain. In 12th IEEE International Conference on Tools with Artificial Intelligence (ICTAI 2000), 13-15 November 2000, Vancouver, BC, Canada, pages 186-189, 2000.

2. Davide Carneiro, Marco Gomes, Paulo Novais, and José Neves. chapter Developing Dynamic Conflict Resolution Models Based on the Interpretation of Personal Conflict Styles, pages 44-58. Springer Berlin Heidelberg, Berlin, Heidelberg, 2011.

3. Finn V. Jensen. Bayesian networks. Wiley Interdisciplinary Reviews: Computational Statistics, 1(3):307-315, 2009.

4. Marco Gomes, Tiago Oliveira, Davide Carneiro, Paulo Novais, and José Neves. Studying the effects of stress on negotiation behavior. Cybernetics and Systems, 45(3):279-291, 2014.

5. Dimitris Margaritis. Learning Bayesian network model structure from data. $\mathrm{PhD}$ thesis, Carnegie-Mellon University, Pittsburgh, 2003.

6. Laura E. Tsamardinos, Ioannisand Brown and Constantin F. Aliferis. The max-min hillclimbing bayesian network structure learning algorithm. Machine Learning, 65(1):31-78, 2006.

7. Marco Scutari. Learning Bayesian networks with the bnlearn R package. Journal of Statistical Software, 35(3):1-22, 2010.

8. Kevin Korb and Ann Nicholson. Bayesian Artificial Intelligence. CRC Press, London, 2 edition, 2011.

9. Ken Satoh, Philippe Codognet, and Hiroshi Hosobe. Speculative constraint processing in multi-agent systems. In Jaeho Lee and Mike Barley, editors, Intelligent Agents and MultiAgent Systems, volume 2891 of Lecture Notes in Computer Science, pages 133-144. Springer Berlin Heidelberg, 2003.

10. T Oliveira, K Satoh, J Neves, and P Novais. Applying Speculative Computation to GuidelineBased Decision Support Systems. In IEEE 27th International Symposium on ComputerBased Medical Systems 2014 (CBMS), pages 42-47, 2014.

11. Robert Castelo and Tomáas Kocka. On inclusion-driven learning of bayesian networks. $J$. Mach. Learn. Res., 4:527-574, December 2003.

12. Ioannis Tsamardinos, Constantin F Aliferis, Alexander R Statnikov, and Er Statnikov. Algorithms for large scale markov blanket discovery. In Proceedings of the Sixteenth International Florida Artificial Intelligence Research Society Conference, volume 2, pages 376-381. AAAI Press, 2003. 